\title{
The roles of evolutionary computation, fitness landscape, constructive methods and local searches in the development of adaptive systems for infrastructure planning
}

\author{
Mehrdad Amirghasemi ${ }^{\mathrm{a}^{*}}$ \\ Reza Zamani ${ }^{\mathrm{a}}$
}

\begin{abstract}
Modelling and systems simulation for improving city planning, and traffic equilibrium are involved with the development of adaptive systems that can learn and respond to the environment intelligently. By employing sophisticated techniques which highly support infrastructure planning and design, evolutionary computation can play a key role in the development of such systems. The key to presenting solution strategies for these systems is fitness landscape which makes some problems hard and some problems easy to tackle. Moreover, constructive methods and local searches can assist evolutionary searches to improve their performance. In this paper, in the context of infrastructure, in general, and city planning, and traffic equilibrium, in particular, the integration of these four concepts is briefly discussed.
\end{abstract}

Key words: Evolutionary computation; Local search; Infrastructure; City planning; Traffic equilibrium.

\section{Introduction}

Infrastructure is a term used to indicate both organizational and physical structures a society or an enterprise needs to function. In effect, all facilities and services needed for the operation of an economy is gathered under the umbrella term of "Infrastructure". Each Infrastructure includes connected elements which are structurally related and each element can affect the other elements. It is through this interconnection that these elements collectively provide a basis for structural development of a society or an enterprise.

Viewed in an operative level, infrastructure makes the production of goods and services required in the society more straightforward and the distribution of finished products to market easier. Whereas these services comprise a wide range from health-care through transportation to education, the produced goods encompass a range from agricultural products through furniture to electronic appliances.

\footnotetext{
${ }^{a}$ SISAT, Faculty of Engineering and Information Sciences, University of Wollongong, NSW 2522, Australia

"Corresponding author: Mehrdad Amirghasemi mehrdad@uow.edu.au http://dx.doi.org/10.14453/isngi2013.proc.2
} 
A better supply of accommodation, water, food, telecommunication, electricity, transportation, and waste disposal, as seven major requirements of a society, all depends on the sophistication of the corresponding infrastructure. Any of these seven major components is either related to providing services, producing goods, or distributing finished products to the points of demand.

Because of the complication of these systems, reaching a reasonable level of efficiency and effectiveness is highly related to employing sophisticated optimization techniques and mathematical programming. This is involved with the optimized selection of a set of values for a number of variables among a set of available alternatives determined by the existing constraints towards minimizing or maximizing a given criterion.

Figures 1 and 2 show a simple and a hard optimization problem, respectively. Because of the complicatedness mentioned, most of the optimization problems in the area of infrastructure belong to the second category. Moreover, each optimization problem can be located in a 3dimensional space with the three axes of time-horizon, number of variables, and probabilistic nature (0-1).

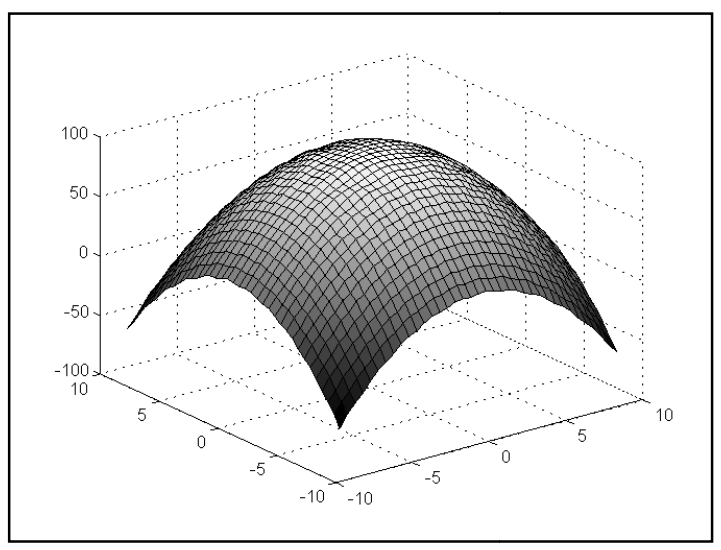

Figure 1. The fitness landscape of an easy optimisation problem.

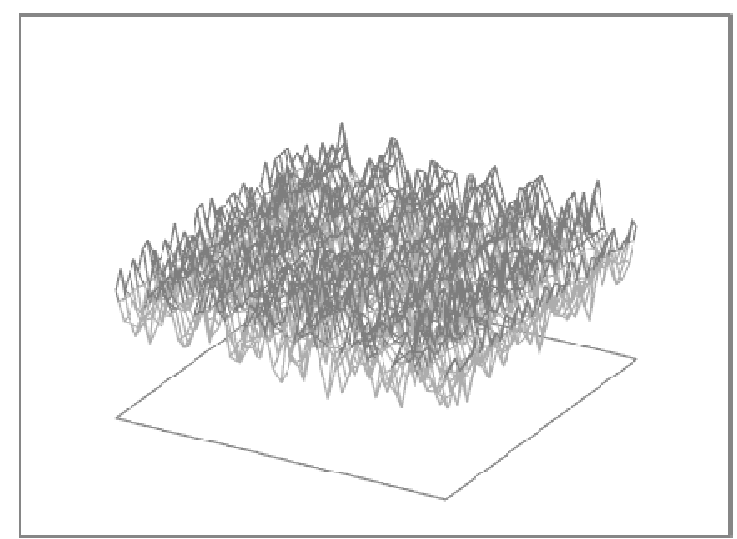

Figure 2. The fitness landscape of a hard optimisation problem. 
Figure 3 shows such a space and that the larger the distance of a problem form the origin $(0,0,0)$, the harder the problem can be. In the area of infrastructure, the number of variables is high, the time-horizon is large, and the probabilistic nature is at the extreme, indicating the tremendous hardness of such problems in comparison of other problems. For instance, the time horizon of such problems is between five to ten years, and these problems are involved with enormous number of variables, which have mainly stochastic nature. Because of such extreme hardness, modelling and simulation of infrastructure problems are complicated issues and are involved with the development of highly effective systems that can learn and respond to the environment intelligently.

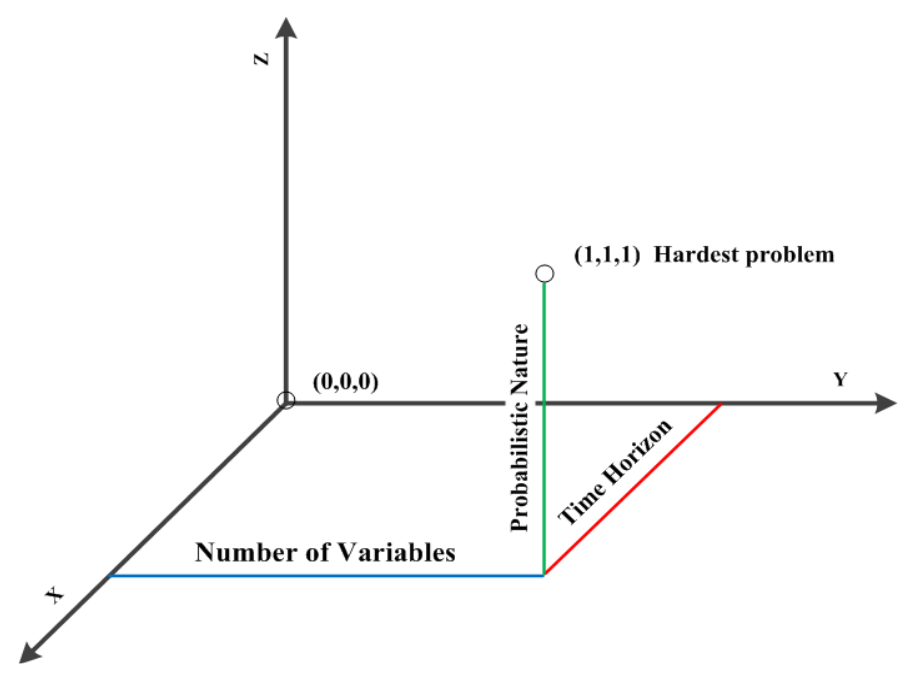

Figure 3. A 3-Dimensional representation of problem difficulty.

With traditional optimization techniques being ineffective or infeasible for such problems, simple agents with limited intelligence can collectively represent complex behaviour and assist infrastructure planning. As a subfield of computational intelligence, and inspired by the biological mechanisms used in nature, genetic algorithms are based on an iterative process which controls the growth and development of a population of solutions competing to survive. The key point with genetic algorithms is the ease of their implementation on massively parallel processors, which makes their solution remarkably easier to obtain.

Using sophisticated techniques and relying on the survival of the fittest solutions, evolutionary computation can highly support infrastructure planning and play a key role in the development of such systems. Despite the fact that genetic algorithm is only one of the three branches of evolutionary computation, and genetic programming and evolution strategies are the two other branches, ${ }^{1}$ in the rest of the paper we use the term genetic algorithms and ignore the other two branches for simplicity.

Despite their robustness, genetic algorithms do not have any fine tuning capability and can be integrated with local searches to remove this shortcoming. Since both local searches and 
evolutionary searches require initial solutions to start, these integrated systems also require an effective constructive method as well. The key to presenting solution strategies for these integrated systems is fitness landscape which makes some problems hard and some problems easy to tackle. The rest of the paper is as follows. Section II briefly discusses the outlines of two infrastructure models with their corresponding references. Section III explains hybrid strategies which include evolutionary search techniques, constructive methods and local searches and also discusses the concept of fitness landscape. Section IV provides concluding remarks and discusses the advantages and disadvantages of evolutionary search techniques for infrastructure problems.

\section{Two examples of highly sophisticated infrastructure models}

In this section, two related infrastructure models are presented and their complexities are discussed. The first model is related to a high-way construction problem and the second model discusses the proper allocation of available regions to residential, commercial, industrial and educational complexes. Examples of educational complexes are universities and TAFE, and examples of commercial complexes are shopping centres and malls. We first start with the highway construction and then continue with the problem of proper allocation of available regions to the complexes.

\section{A. Traffic equilibrium and highway construction}

City Transportation is the movement of people from one location of the city to another. The main modes of transport are roads, streets, highways and railways. In a city, transportation infrastructure is as important as a telecommunication infrastructure, because it enables personal communication among people, which in turn facilitates daily work. Decisions about the improvement of transportation infrastructure, however, are among the most complex decisions. Part of this complexity is because of the extreme interplay of variables with one another. For instance, establishing a highway between the two points of "a" and "b" affects the load of traffic in points much further than these two points. Exactly the same as a succession of processes such as the components of the electrical circuits and chemical reactions, in which each element activates other elements, all elements of transportation affect the other elements to a considerable extent.

This succession of processes makes the construction of new highways as one of the most expensive activities in improving an urban infrastructure. When a budget is assigned to highway construction projects, the main question is that to which highways this budget should be assigned. The answer to this question depends on how different ways of spending this budget changes the traffic flow. Traffic flow itself is the study of interactions among drivers to select their path to reach their destination from the origin they have started in the shortest possible time. The decision of drivers not only depends on the decision of other drivers but also on the available streets, roads and highways. Depending on some particular spending of such a budget, some drivers may also switch from using their private car to public transportation.

The study of this complicated system and the way passengers, in general, and drivers, in particular, connect their origin to their destination highly affects the development of an optimal road network. In effect, without sophisticated optimization techniques in assigning budgets to the 
development of highways, efficient movement of traffic is near to impossible. Figure 4 shows a simplified model for spending a certain budget for developing a number of possible highways. Interested reader may refer to the related studies in road network design for more info. ${ }^{2}$

1. Find the interplay among variables through Origin / Destination data of traffic;

2. Considering the problem constraints, test possible scenarios for highway construction through calculating average time spent in traffic and cost;

3. Select the best alternatives with respect to the total budget;

4. Decide about the highways to be constructed based on the allocated budget.

Figure 4. A simplified model for spending a certain budget for developing a number of possible highways

\section{B. City planning through allocation of regions to complexes}

City planning is a technical process corresponding to the design of the urban environment to ensure an orderly development of settlement. Extremely concerned with analytical issues and systems planning, it can take a variety of forms and its output is involved with the systematic recommendation about the installation of different complexes, from libraries through shopping centres to recreational centres, in different locations of the cities. The possible construction of a site, depending on whether it is residential or non-residential, causes the production or attraction of many different trips. Non-residential complexes can be educational, commercial, industrial, recreational, or medical, with each of these types of complexes absorbing particular trips.

Assume that there are $\mathrm{n}$ sites and $\mathrm{m}$ complexes to be built. The strategic infrastructure question is which site should be allocated to each complex. In effect, such a question is concerned with a very large search space and is involved with highly sophisticated modelling and computer programming. A mathematical model for allocating proper sites to possible complexes is highly multifaceted. Figure 5 shows a simplified model for the allocation of regions to complexes. Interested reader may refer to related studies. ${ }^{3}$

1 Find the interplay among variables through Origin / Destination data of traffic;

2. Considering the problem constraints, test possible scenarios for assigning; regions to complexes through calculating average time spent in traffic and cost;

3. Select the best alternatives;

4. Decide about allocating regions to related complexes.

Figure 5. A simplified model for the allocation of regions to complexes 
As is seen, there are several key points in the commonality and differences of the two aforementioned models. First, both models use the current Origin / Destination data and extend it to the future, when the construction of the project is completed, whether it is the construction of the highways or the complexes. Second, both models have the same objective function of minimizing average time spent in traffic. Third, in the first model, the decision variables comprise a vector of size $\mathrm{n}$, in which $\mathrm{n}$ shows the number of highways that can be constructed whereas in the second model, the decision variables establish an $\mathrm{m} \times \mathrm{n}$ matrix, where $\mathrm{m}$ and $\mathrm{n}$ show the number of available locations and complexes, respectively.

\section{Evolutionary search techniques, constructive methods, local searches and fitness landscape}

In solving the problem of city planning through allocation of regions to complexes, a building block is as follows. Given a set of $n$ facilities and $n$ locations, and two $n \times n$ matrices, one representing the flow between each two facilities, $\mathrm{f}_{\mathrm{ij}}$, and the other representing the distance between each two locations $d_{k l}$, the goal is to find an arrangement $\pi$ for the facilities that minimizes the volume of traffic among the facilities. This is an old and notorious problem in optimization called facility-layout problem. In the optimization literature, some researchers have also called it Quadratic Assignment Problem. ${ }^{4}$ Based on this notorious problem, the traffic volume is the sum of all possible distance-flow products.

For this problem, the most effective meta-heuristic procedure can be classified under three main categories of constructive methods, local searches and genetic algorithms. In constructive methods, a solution is constructed step-by-step. In fact, solution elements are determined at each iteration based on a heuristic procedure. On the other hand in local searches a complete solution is improved by iteratively applying certain operators such as swapping the location of two facilities. In the third category, genetic algorithms, a population of solutions are collectively evolved via competition and cooperation of individual solutions through selection and recombination operators, respectively. ${ }^{5}$

The evolutionary search procedures for tackling this problem adaptively employ the three mentioned search paradigms and can be illustrated by Figure 6. In fact, first, a constructive procedure is used to build an initial pool of high quality solutions. Next, an enhancement procedure is applied to all individual solutions in the pool for further improvement. Finally, the genetic algorithm tries to produce higher quality solutions (offspring) by combining the current enhanced solutions (parents). The success of these hybrid procedures in tackling combinatorial optimization problems is the main motivation in using adaptive, hybrid meta-heuristics for complicated infrastructure problems.

Based on "No-free-lunch" theorem grounds, ${ }^{6}$ incorporating problem-specific knowledge, and matching the "procedure" with the "problem" is essential to develop high performance problem solvers. Nevertheless, the ideal case is to have a procedure as generic as possible to solve a class of similar problems effectively. To balance this dilemma, we require the proposed model to be as modularized as possible, separating the problem-specific parts from the generic parts. 
The other concern in the development of efficient meta-heuristic procedures is the notion of fitness-distance correlation in the search landscape. For instance, using solutions obtained from a constructive procedure as initial solutions as an input solution for other modules, significantly increases the quality of the final output solution. This improvement in solution quality is amplified when the fitness-distance correlation is high and the problem search landscape has a "big valley" structure. ${ }^{7-8}$

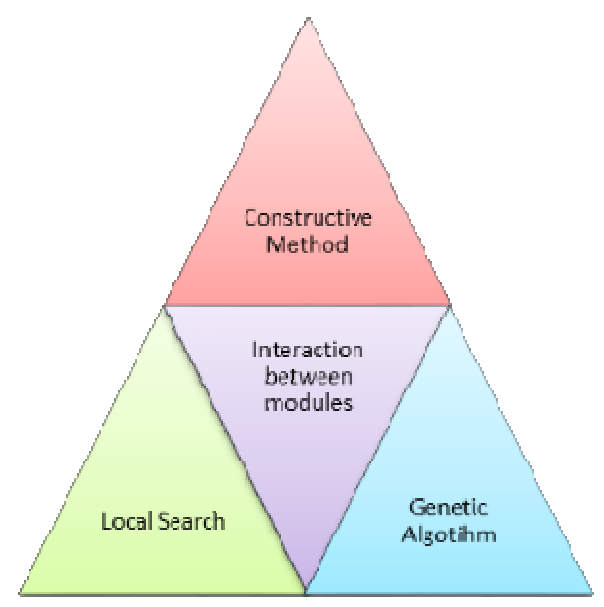

Figure 6. The modularisation of the hybrid models for infrastructure problems

\section{Concluding remarks}

Modelling and simulation of infrastructure problems are complicated issues and require a sophisticated solution methodology and scientific analysis. That is why without scientific analysis of a city and its surroundings and without having an intricate solution methodology, the planning of the extension of the city leads to serious issues. A laborious project concerned with the scientific analysis of the city requires many technical details and the result can be represented as a model. These models are highly complicated and because of their involvement with a wide variety of interacting variables, cannot be solved easily. Physical processes on which the history of life is explained can be imitated to tackle these hard-to-solve problems.

The main notions of such a solution methodology are mutation, reproduction, and most importantly the survival of the fittest. For several reasons, genetic algorithms are of key importance in solving the models presented in this paper. First, as a population-based approach, genetic algorithms are intrinsically parallel and this facilitates the solution of many hard-to-solve infrastructure problems. Second, they are highly adaptive and flexible and this contributes to their wide applicability. Third, they can operate in large search-spaces effectively, and fourth, they are easy to implement. An evolutionary search technique maintains a population of solutions, impose random changes to these solutions mainly through mutation and crossover operations and applies the survival of the fittest principal to the pool of solutions, with a 
selection process directing which solutions can participate in crossover operations and which survives for the next pool.

Without using construction methods and local searches, the application of genetic algorithms, however, cannot produce high quality results. Hence the integration of construction methods, local searches and genetic algorithms seems to be an effective approach in dealing with these highly complicated problems. Fitness landscape of these problems can determine how this integration can best be performed.

In both of the presented models, the origin-destination data were used. Origin-destination surveys can be simply performed in different cities to determine the transportation needs. The typical outputs of these systems are several matrices, with each matrix related to a different purpose of traffic in different hours of the day for different modes of transportation. Each cell $(\mathrm{i}, \mathrm{j})$ of such matrices shows the number of trips from origin $\mathrm{i}$ to destination $\mathrm{j}$. In other words, before such a study, the city should be divided into a number of zones. For instance, if the city is divided into 500 zones, then all these matrices are of the size $500 \times 500$, which is computationally demanding. The understanding of the underlying characteristics of travel is, however, only possible through the study and manipulation of these matrices.

\section{References}

${ }^{1}$ Fogel, D. B., "An introduction to simulated evolutionary optimization," IEEE Transactions on Neural Networks, Vol. 5, No. 1, 1994, pp. 3-14. http://dx.doi.org/10.1109/72.265956

${ }^{2}$ Szeto, W. Y., Jiang, Y., Wang, D. Z. W., and Sumalee, A. "A sustainable road network design problem with land use transportation interaction over time," Networks and Spatial Economics, June 2013, pp. 1-32.

${ }^{3}$ Larice, M., and Macdonald, E. (Editors), The urban design reader. Routledge, 2013.

${ }^{4}$ Burkard, R. E. "Quadratic assignment problems", European Journal of Operational Research, Vol. 15, No.3, 1984, pp. 283-289. http://dx.doi.org/10.1016/0377-2217(84)90093-6

${ }^{5}$ De Jong, K. A., Evolutionary computation: a unified approach, MIT Press, 2006.

${ }^{6}$ Wolpert, D. H., and W. G. Macready, "No free lunch theorems for optimization," IEEE Transactions on Evolutionary Computation, Vol. 1, No. 1, 1997, pp. 67-82. http://dx.doi.org/10.1109/4235.585893

${ }^{7}$ Boese, K. D., A. B. Kahng, et al., "A new adaptive multi-start technique for combinatorial global optimizations," Operations Research Letters, Vol. 16, No. 2, 1994, pp. 101-113. http://dx.doi.org/10.1016/0167-6377(94)90065-5

${ }^{8}$ Jones, T. and Forrest S., Fitness Distance Correlation as a Measure of Problem Difficulty for Genetic Algorithms. Proceedings of the 6th International Conference on Genetic Algorithms, Morgan Kaufmann Publishers Inc., 1995, pp. 184-192. 